TRANSACTIONS OF THE

AMERICAN MATHEMATICAL SOCIETY

Volume 354, Number 7, Pages 2745-2758

S 0002-9947(02)02993-8

Article electronically published on March 7, 2002

\title{
REPRESENTATIONS OF EXCEPTIONAL SIMPLE ALTERNATIVE SUPERALGEBRAS OF CHARACTERISTIC 3
}

\author{
M. C. LÓPEZ-DÍAZ AND IVAN P. SHESTAKOV
}

\begin{abstract}
We study representations of simple alternative superalgebras $B(1,2)$ and $B(2,4)$. The irreducible bimodules and bimodules with superinvolution over these superalgebras are classified, and some analogues of the Kronecker factorization theorem are proved for alternative superalgebras that contain $B(1,2)$ and $B(4,2)$.
\end{abstract}

\section{INTRODUCTION}

The simple alternative superalgebras were classified in [6] and [5]. In particular, it was proved in [5] that a simple alternative superalgebra $B=B_{0}+B_{1}$, which is not just a $Z_{2}$-graded alternative algebra, should necessarily have characteristic 3 and be isomorphic to one of the following superalgebras over a field $F$ of characteristic 3 .

1) $B=B(1,2)$, where $B_{0}=F \cdot 1, B_{1}=F \cdot x+F \cdot y$, with 1 being the unit of $B$ and $x y=-y x=1, x^{2}=y^{2}=0$.

2) $B=B(4,2)$, where $B_{0}=M_{2}(F), B_{1}=F \cdot m_{1}+F \cdot m_{2}$ is the 2-dimensional irreducible Cayley bimodule over $B_{0}$; that is, $B_{0}$ acts on $B_{1}$ by

$$
\begin{aligned}
e_{i j} \cdot m_{k} & =\delta_{i k} m_{j}, \quad i, j, k \in\{1,2\}, \\
m \cdot a & =\bar{a} \cdot m,
\end{aligned}
$$

where $a \in B_{0}, m \in B_{1}, a \rightarrow \bar{a}$ is the symplectic involution in $B_{0}=M_{2}(F)$. The odd multiplication on $B_{1}$ is defined by

$$
m_{1}^{2}=-e_{21}, m_{2}^{2}=e_{12}, m_{1} m_{2}=e_{11}, m_{2} m_{1}=-e_{22} .
$$

3) The twisted superalgebra of vector type $B=B(E, D, \gamma)$. Let $E$ be a commutative and associative algebra over $F, D$ be a nonzero derivation of $E$ such that $E$ is $D$-simple, and $\gamma \in E$. Denote by $\bar{E}$ an isomorphic copy of the vector space $E$, with an isomorphism mapping $a \rightarrow \bar{a}$. Consider the vector space direct sum $B(E, D, \gamma)=E+\bar{E}$ and define multiplication on it by the rules

$$
a \cdot b=a b, \quad a \cdot \bar{b}=\bar{a} \cdot b=\overline{a b}, \quad \bar{a} \cdot \bar{b}=\gamma a b+2 D(a) b+a D(b),
$$

Received by the editors April 17, 2001.

2000 Mathematics Subject Classification. Primary 17D05, 17A70, 17C70.

Key words and phrases. Alternative superalgebra and superbimodule, superinvolution, factorization theorem.

The first author was supported by FAPESP 2000/03404-5 and FICYT PB-PGI 99-04.

The second author was supported by CNPq grant 300528/99-0.

(C)2002 American Mathematical Society 
where $a, b \in E$ and $a b$ is the product in $E$. A $Z_{2}$-grading on $B=B(E, D, \gamma)$ is defined by $B_{0}=E$ and $B_{1}=\bar{E}$. In any characteristic, $B$ is a simple right alternative superalgebra; and when $\operatorname{char} F=3, B$ is alternative.

In this work, we study birepresentations of $B(1,2)$ and of $B(4,2)$. First, we classify the irreducible superbimodules over these superalgebras. It occurs that, besides a certain two-parametric series of bimodules $V(\lambda, \mu)$ over $B(1,2)$, all the other unital irreducible superbimodules for these superalgebras are regular or opposite to them. As a corollary, we prove that every unital $B(4,2)$-superbimodule is completely reducible. Besides, every alternative superalgebra $B$ that contains $B(4,2)$ as a unital subsuperalgebra admits a graded Kronecker factorization $B=B(4,2) \widetilde{\otimes} U$ for a certain associative commutative superalgebra $U$.

It was shown in [5] that both $B(1,2)$ and $B(4,2)$ admit $J$-admissible superinvolutions; that is, superinvolutions with symmetric elements in the nucleus. This was used in [5] for constructing new simple exceptional Jordan superalgebras of characteristic 3 as $3 \times 3$ Hermitian matrices over $B(1,2)$ and $B(4,2)$. Motivated by the future study of representations of these Jordan superalgebras, we classify the irreducible bimodules with $J$-admissible superinvolution over $B(1,2)$ and $B(4,2)$. In the case of $B(4,2)$, the list of irreducible bimodules with superinvolution coincides with that of irreducible bimodules, and for $B(1,2)$ this list contains only regular supermodules and their opposites, while the supermodules $V(\lambda, \mu)$ do not enter in the list. As a corollary, every unital supermodule with $J$-admissible superinvolution over $B(1,2)$ is completely reducible; and every alternative superalgebra with $J$-admissible superinvolution that contains $B(1,2)$ as a unital subsuperalgebra admits a Kronecker factorization as above.

Now, let us recall some definitions and fix certain notation.

A superalgebra $A=A_{0}+A_{1}$ over a field $F$ is called alternative if it satisfies the superidentities

$$
(x, y, z)=-(-1)^{d(x) d(y)}(y, x, z)=-(-1)^{d(y) d(z)}(x, z, y),
$$

where $(x, y, z)=(x y) z-x(y z), \quad x, y, z \in A_{0} \cup A_{1}$, and $d(r)$ stands for the parity index of a homogeneous element $r: d(r)=i$ if $r \in A_{i}$. In this case, it is easy to see that $A_{0}$ is an alternative algebra and $A_{1}$ is an alternative bimodule over $A_{0}$.

An $A$-superbimodule $M=M_{0}+M_{1}$ is called an alternative superbimodule if the corresponding split extension superalgebra $E=A+M$ is alternative.

For an $A$-superbimodule $M$, the opposite superbimodule $M^{o p}=M_{0}^{o p}+M_{1}^{o p}$ is defined by the conditions $M_{0}^{o p}=M_{1}, M_{1}^{o p}=M_{0}$, and the following action of $A$ : $a \cdot m=(-1)^{d(a)} a m, m \cdot a=m a$, for any $a \in A_{0} \cup A_{1}, m \in M^{o p}$. If $M$ is an alternative $A$-superbimodule, then one can easily check that so is $M^{o p}$.

A regular superbimodule, $\operatorname{Reg} A$, for a superalgebra $A$, is defined on the vector superspace $A$ with the action of $A$ coinciding with the multiplication in $A$.

We will denote, for any homogeneous $a$ and $b$,

$$
\begin{aligned}
& {[a, b]:=a b-(-1)^{d(a) d(b)} b a,} \\
& a \circ b:=a b+(-1)^{d(a) d(b)} b a .
\end{aligned}
$$

If not stated otherwise, throughout the paper $F$ will denote a field of characteristic 3. All the algebras and superalgebras will be considered over $F$. 


\section{Representations of $B(1,2)$}

In this section, we classify irreducible superbimodules over the superalgebra $B(1,2)$, defined in the Introduction.

We start with the following general result.

Proposition 2.1. Let $B$ be a simple commutative non-associative alternative superalgebra, and let $V$ be an irreducible alternative $B$-superbimodule. Then $V$ is commutative, that is, for any $v \in V_{0} \cup V_{1}, a \in B_{0} \cup B_{1},[v, a]=0$ holds.

Proof. Let us show first that for any homogeneous $a \in B$ the set $[V, a]:=\{[v, a] \mid v \in$ $V\}$ forms a subbimodule of $V$. Recall two identities that are valid in alternative superalgebras (see [5, 7]):

$$
\begin{aligned}
{[x y, z]-x[y, z]-(-1)^{d(y) d(z)}[x, z] y-3(x, y, z) } & =0 \\
{[[x, y], z]-(-1)^{d(y) d(z)}[[x, z], y]-[x,[y, z]]-6(x, y, z) } & =0 .
\end{aligned}
$$

Since $B$ is commutative and $\operatorname{char} B=3$, we have by (3) for any homogeneous $v \in V, b \in B$

$$
\begin{aligned}
& {[v, a] b=(-1)^{d(a) d(b)}[v b, a],} \\
& b[v, a]=[b v, a],
\end{aligned}
$$

which proves that $[V, a]$ is a subbimodule of $V$. Assume that there exists $z \in B_{1}$ such that $[V, z] \neq 0$. Then, by irreducibility, $V=[V, z]=[[V, z], z]$. But it follows from (4) that $[[v, z], z]=-[[v, z], z]=0$; hence $V=[[V, z], z]=0$, a contradiction. Therefore,

$$
\left[V, B_{1}\right]=0 .
$$

Now, the set $B_{1}+B_{1}^{2}$ is an ideal in $B$. If it were zero, then $B=B_{0}$ would be a field; so we have $B=B_{1}+B_{1}^{2}$. Let $x, y \in B_{1}, v \in V$. Then we have by (3)

$$
[x y, v]=x[y, v]+(-1)^{d(v)}[x, v] y=0 .
$$

Thus, $[B, V]=0$, proving the proposition.

Corollary 2.1. Every unital alternative superbimodule $V$ over the superalgebra $B=B(1,2)$ satisfies the condition

$$
[[[V, B], B], B]=0 \text {. }
$$

Proof. It was proved above that, for any $v \in V, z \in B_{1}$, the equality $[[v, z], z]=0$ holds. Linearizing it, we have $[[v, x], y]=-[[v, y], x]$. In particular, $[[V, B], B]=$ $[[V, x], y]=[[V, y], x]$. Therefore,

$$
[[[V, B], B], x]=[[[V, y], x], x]=0,
$$

and similarly $[[[V, B], B], y]=0$, proving the corollary.

Denote by $V(\lambda, \mu)$, for $\lambda, \mu \in F$, the commutative superbimodule over $B(1,2)=$ $F \cdot 1+F \cdot x+F \cdot y$, with the basis

$$
v_{0}, v_{1} y, v_{0} y^{2} \text { for } V_{0}, v_{1}, v_{0} y, v_{1} y^{2} \text { for } V_{1},
$$

and the action of $x$ and $y$ defined as follows. Let $v$ stand for any of the elements $v_{0}, v_{1}$ and $v_{i}^{s}=v_{1-i}$. Then

$$
\begin{array}{r}
v y^{j} \cdot y=v y^{j+1}, \quad j=0,1 ; \quad v y^{2} \cdot y=\mu v^{s} \\
v y^{j} \cdot x=\lambda v^{s} y^{j}+j v y^{j-1}, \quad j=0,1,2 .
\end{array}
$$


Proposition 2.2. The superbimodule $V(\lambda, \mu)$ is alternative for any $\lambda, \mu$ and irreducible if $\lambda \neq 0$ or $\mu \neq 0$.

Proof. It is easy to see that in any commutative superalgebra the equality

$$
(a, b, c)=-(-1)^{d(a) d(b)+d(a) d(c)+d(b) d(c)}(c, b, a)
$$

holds. This implies easily that every right alternative commutative superbimodule over a commutative superalgebra is also left alternative. Hence, it suffices to prove that $V(\lambda, \mu)$ is right alternative. For this we need to check the following identities:

$$
\begin{aligned}
& (u, x, y)-(u, y, x)=0, \\
& (x, u, y)+(-1)^{d(u)}(x, y, u)=0, \\
& (y, u, x)+(-1)^{d(u)}(y, x, u)=0, \\
& (x, u, x)+(-1)^{d(u)}(x, x, u)=0, \\
& (y, u, y)+(-1)^{d(u)}(y, y, u)=0,
\end{aligned}
$$

where $u$ is any element of the base. Let us start with (5). For $u=v y^{j}, j=0,1$, we have

$$
\begin{aligned}
& \left(v y^{j}, x, y\right)=\lambda v^{s} y^{j+1}+(j-1) v y^{j}, \\
& \left(v y^{j}, y, x\right)=\lambda v^{s} y^{j+1}+(j+1) v y^{j}+v y^{j},
\end{aligned}
$$

which gives (5) since $\operatorname{char} F=3$. Similarly,

$$
\begin{aligned}
& \left(v y^{2}, x, y\right)=\lambda \mu v+2 v y^{2}-v y^{2}, \\
& \left(v y^{2}, y, x\right)=\mu v^{s} \cdot x+v y^{2}=\mu \lambda v+v y^{2},
\end{aligned}
$$

which proves (5).

Furthermore, by commutativity,

$$
\begin{aligned}
& (x, u, y)=(-1)^{d(u)}(u x \cdot y+u y \cdot x), \\
& (x, y, u)=u \cdot x y+u y \cdot x
\end{aligned}
$$

hence $(x, u, y)+(-1)^{d(u)}(x, y, u)=(-1)^{d(u)}(u x \cdot y+u y \cdot x+u \cdot x y+u y \cdot x)=$ $(-1)^{d(u)}(u x \cdot y-u y \cdot x-u \cdot x y+u \cdot y x)=(-1)^{d(u)}((u, x, y)-(u, y, x))=0$ by (5). Similarly, we have (7). Finally, we have

$$
\begin{aligned}
& (x, u, x)=(-1)^{d(u)}(u x \cdot x+u x \cdot x), \\
& (x, x, u)=u x \cdot x,
\end{aligned}
$$

which proves (8) and, similarly, (9). Hence, the module $V(\lambda, \mu)$ is alternative. One can easily check that if $\lambda \neq 0$ or $\mu \neq 0$, then this module is irreducible.

Observe that the opposite bimodule $(V(\lambda, \mu))^{o p}$ is isomorphic to $V(\lambda, \mu)$ under the isomorphism $v y^{j} \mapsto v^{s} y^{j}$. It is also easy to see that the modules $V(\lambda, \mu)$ and $V\left(\lambda^{\prime}, \mu^{\prime}\right)$ are isomorphic if and only if $(\lambda, \mu)= \pm\left(\lambda^{\prime}, \mu^{\prime}\right)$.

Theorem 2.1. Every irreducible unital alternative superbimodule $V$ over $B(1,2)$, in the case where the ground field $F$ (of characteristic 3) is algebraically closed, is isomorphic to one of the bimodules: $\operatorname{Reg} B(1,2),(\operatorname{Reg} B(1,2))^{o p}, V(\lambda, \mu) \mathbf{1}$

\footnotetext{
${ }^{1} \mathrm{~V}$. N. Zhelyabin informed the authors that a classification of irreducible alternative superbimodules over $B(1,2)$ was also obtained by M. Trushina.
} 
Proof. According to Proposition 2.1, we can assume that $V$ is commutative; so we may restrict ourselves to considering only the right actions $\rho(x)$ and $\rho(y)$ of $x$ and $y$ on $V$. Let us prove first that the elements $\rho(x)^{3}$ and $\rho(y)^{3}$ lie in the centralizer of $V$ as a right $B(1,2)$-module.

We will use in this proof non-graded (ordinary) commutators, which we will denote by

$$
[a, b]_{0}:=a b-b a,
$$

in order to distinguish them from the graded commutators, defined in the Introduction. By super-rightalternativity, we have for any $v \in V$

$$
(v x) y-(v y) x=v(x y-y x)=2 v=-v,
$$

which gives

$$
[\rho(x), \rho(y)]_{0}=-i d_{V}
$$

Now

$$
\begin{aligned}
{\left[\rho(x)^{3}, \rho(y)\right]_{0} } & =\rho(x)^{2}[\rho(x), \rho(y)]_{0}+[\rho(x), \rho(y)]_{0} \rho(x)^{2}+\rho(x)[\rho(x), \rho(y)]_{0} \rho(x) \\
& =-3 \rho(x)^{2}=0
\end{aligned}
$$

Thus $\rho(x)^{3}$ lies in the centralizer of $V$, and similarly so does $\rho(y)^{3}$.

Consider the two possible cases separately.

$1^{\circ} \cdot \rho(x)^{3}=\rho(y)^{3}=0$.

Let us prove that in this case $V$ is isomorphic to $\operatorname{Reg} B(1,2)$ or to its opposite bimodule. Observe first that $\rho(x)^{2} \neq 0$. In fact, we have by (10)

$$
\left[\rho(x)^{2}, \rho(y)\right]_{0}=\rho(x)[\rho(x), \rho(y)]_{0}+[\rho(x), \rho(y)]_{0} \rho(x)=-2 \rho(x) ;
$$

so $\rho(x)^{2}=0$ would imply $\rho(x)=0$, which is impossible. Assume that $\left.\rho(x)^{2}\right|_{V_{i}} \neq 0$ for some $i \in\{0,1\}$, that is, there exists $v \in V_{i}$ such that $u=(v x) x \neq 0, u \in V_{i}$. Then we have

$$
u x=((v x) x) x=0 .
$$

Observe that, by (10), $(u x) y-(u y) x=-u \neq 0$; hence $u y \neq 0$. Furthermore,

$$
\begin{aligned}
(u y) x & =(u x) y+u=u, \\
((u y) y) y & =0, \\
((u y) y) x & =u y+((u y) x) y=u y+u y=-u y .
\end{aligned}
$$

Therefore, the elements $u, u y,(u y) y$ span a $B(1,2)$-submodule of $V$, which, by irreducibility, coincides with $V$. It it easy to check that if $i=0$, then $V \cong$ $(\operatorname{Reg} B(1,2))^{o p}$, and if $i=1$, then $V \cong \operatorname{Reg} B(1,2)$.

$2^{\circ} . \rho(x)^{3} \neq 0$.

We claim that in this case $V$ is isomorphic to a module of the type $V(\lambda, \mu)$. Let $A=\operatorname{alg}_{F}\langle\rho(x), \rho(y)\rangle$ be a subalgebra of $\operatorname{End}_{F} V$ generated by $\rho(x), \rho(y)$. Since $V$ is irreducible, the center $Z=Z(A)$ is a graded division algebra; besides, $Z_{1} \ni$ $\rho(x)^{3} \neq 0$. It is easy to see that in this case $Z=Z_{0}+Z_{0} s$ for any fixed $0 \neq s \in Z_{1}$; in particular, $\rho(x)^{3}=\alpha s, \rho(y)^{3}=\mu s$ for some $\alpha, \mu \in Z_{0}$. Let $E=\operatorname{alg}_{F}\left\langle\alpha, \mu, s^{2}\right\rangle$. Then $E \subseteq Z_{0}$ and $A$ is spanned over $E$ by the elements $\rho(x)^{i} \rho(y)^{j}, s \rho(x)^{i} \rho(y)^{j}, 0 \leq$ $i, j \leq 2$. In particular, $V$ is finite dimensional over $Z_{0}$. Since $V$ is a commutative supermodule, by [1, Proposition 4.2], it is irreducible as an ordinary (non-graded) $A$-module. This implies, by the density theorem, that $A=E n d_{Z_{0}} V$. Let us show that $Z_{0}=E$. Consider some $z \in Z_{0}, z=\alpha_{0}+\alpha_{1} \rho(y)+\alpha_{2} \rho(y)^{2}$, where $\alpha_{i}$ depend 
only on $\rho(x)$ and $s$. We have $0=[z, \rho(x)]_{0}=\alpha_{1}+2 \alpha_{2} \rho(y)$. Multiplying this by $\rho(y)$ and subtracting from $z$, we get $z=\alpha_{0}-\alpha_{2} \rho(y)^{2}$. Commuting $z$ with $\rho(x)$ again, we get $\alpha_{2} \rho(y)=0$ and so $z=\alpha_{0}=\beta_{0}+\beta_{1} \rho(x)+\beta_{2} \rho(x)^{2}$, where $\beta_{0}, \beta_{2} \in E, \beta_{1} \in E s$. Commuting now $z$ with $\rho(y)$ and arguing as before, we obtain finally that $z=\beta_{0} \in E$.

Thus, the field $Z_{0}$ is a finitely generated algebra over $F$. Since $F$ is algebraically closed, this implies that $Z_{0}=F$. We can now choose $s \in Z_{1}$ such that $s^{2}=1$. Let $0 \neq \lambda \in F$ be a root of the polynomial $X^{3}-\alpha$ and $v \in V$ such that $s \rho(x)(v)=$ $v^{s} \cdot x=\lambda v$. We can assume, without loss of generality, that $v=v_{0} \in V_{0}$. Denote $v_{1}:=v^{s}, \rho(y)^{j}\left(v_{i}\right):=v_{i} y^{j}$ for $0 \leq j \leq 2$. Then we have

$$
\begin{aligned}
& v_{0} \cdot x=\lambda v_{1}, v_{1} \cdot x=\lambda v_{0} \\
& v_{i} y^{j} \cdot y=v_{i} y^{j+1}, j<2 ; \quad v_{i} y^{2} \cdot y=v_{i} \rho(y)^{3}=\mu v_{1-i} ; \\
& v_{i} y \cdot x=v_{i}[\rho(y), \rho(x)]_{0}+\left(v_{i} \cdot x\right) \cdot y=v_{i}+\lambda v_{1-i} y \\
& v_{i} y^{2} \cdot x=v_{i} y[\rho(y), \rho(x)]_{0}+\left(v_{i} y \cdot x\right) \cdot y=v_{i} y+\lambda v_{1-i} y^{2}+v_{i} y=\lambda v_{1-i} y^{2}+2 v_{i} y .
\end{aligned}
$$

These relations show that $V$ is a homomorphic image of the module $V(\lambda, \mu)$. In order to prove that $V$ is isomorphic to $V(\lambda, \mu)$, it suffices to prove that the elements $v_{0}, v_{1} y, v_{0} y^{2}$ are linearly independent over $F$. It is easy to see that they are nonzero. Assume that

$$
\alpha v_{0}+\beta v_{1} y+\gamma v_{0} y^{2}=0
$$

for some $\alpha, \beta, \gamma \in F$. Applying $s$ to this equality, we get

$$
\alpha v_{1}+\beta v_{0} y+\gamma v_{1} y^{2}=0 .
$$

On the other hand, multiplying (11) by $x$, we get

$$
\alpha \lambda v_{1}+\beta\left(\lambda v_{0} y+v_{1}\right)+\gamma\left(\lambda v_{1} y^{2}+2 v_{0} y\right)=0,
$$

which, by (12), gives

$$
\beta v_{1}+2 \gamma v_{0} y=0 .
$$

Applying $s$ to (13), we get $\beta v_{0}+2 \gamma v_{1} y=0$, and multiplying (13) by $x$, we obtain

$$
0=\beta \lambda v_{0}+2 \gamma\left(\lambda v_{1} y+v_{0}\right)=\lambda\left(\beta v_{0}+2 \gamma v_{1} y\right)+2 \gamma v_{0}=2 \gamma v_{0} .
$$

Thus $\gamma=0$, which implies easily that $\beta=\alpha=0$ as well. This finishes the proof of the theorem.

\section{Representations of $B(4,2)$}

We will use in this section certain results about alternative bimodules over composition algebras that were proved in [5]. For the convenience of the reader, we state these results below.

Recall that a bimodule $V$ over a composition algebra $C$ is called a Cayley bimodule if it satisfies the relation

$$
a v=v \bar{a},
$$

where $a \in C, v \in V$, and $a \rightarrow \bar{a}$ is the canonical involution in $C$. 
Proposition 3.1 ([5] Lemma 11 and its proof $]$ ). Let $B=B_{0}+B_{1}$ be a unital alternative superalgebra over a field $F$ which contains an even composition subalgebra $C$ with the same unit. Assume that a subspace $V$ of $B$ is $C$-invariant and satisfies (14). Then, the following identities hold for any $a, b \in C, r \in B, u, v \in V$.

$$
\begin{aligned}
(a b) v & =b(a v), v(a b)=(v b) a \\
a(u r) & =u(\bar{a} r) \\
a(u v) & =u(v a),(u v) a=(a u) v \\
(u, v, a) & =[u v, a] .
\end{aligned}
$$

Proposition 3.2 ([5 Lemma 12 and its proof $]$ ). Let $H$ be a generalized quaternion algebra. Then, any unital alternative $H$-bimodule $V$ admits the decomposition $V=V_{a} \oplus V_{c}$, where $V_{a}$ is an associative $H$-bimodule and $V_{c}$ is a Cayley bimodule over $H$; moreover, the subbimodule $V_{c}$ coincides with the subspace $(V, H, H)$.

In this section we are going to prove the following theorems which describe the alternative superbimodules over the superalgebra $B(4,2)$.

Theorem 3.1. Let $V$ be a unital irreducible alternative superbimodule over $B(4,2)$. Then $V$ is isomorphic to $\operatorname{Reg}(B(4,2))$ or to $\operatorname{Reg}(B(4,2))^{\text {op }}$.

Theorem 3.2. Every unital alternative superbimodule over $B(4,2)$ is completely reducible.

We divide the proof into a sequence of lemmas.

Let $B=B(4,2)=H+M$, with $H=M_{2}(F), M=F \cdot m_{1}+F \cdot m_{2}$, the 2-dimensional Cayley $H$-bimodule defined by (1) and (2), and let $V$ be a unital irreducible alternative superbimodule over $B$. By Proposition $3.2 V=V_{a} \oplus V_{c}$ where $V_{a}$ is an associative $H$-bimodule and $V_{c}$ is a Cayley $H$-bimodule.

Lemma 3.1. Let $V=V_{a} \oplus V_{c}$ be a unital alternative superbimodule over $B(4,2)=$ $H+M$. Then, for any $v \in V_{c}, m \in M, a \in H$,

$$
\begin{aligned}
& (v m) a=(a v) m, \\
& (m v) a=(a m) v,
\end{aligned}
$$

and for any $u \in V_{a}, m \in M, a, b \in H$,

$$
\begin{aligned}
(u m) a & =(u \bar{a}) m, \\
a(m u) & =m(\bar{a} u), \\
((u m) a) b & =(u m)(b a), \\
b(a(m u)) & =(a b)(m u), \\
(u m, a, b) & =(u m)[b, a], \\
(b, a, m u) & =[b, a](m u) .
\end{aligned}
$$

Proof. First, consider $v \in V_{c}, m \in M, a \in H$. By (14), (vm) $a-(a v) m=(v m) a-$ $(v \bar{a}) m=(v, m, a)-(v, \bar{a}, m)+v(m a-\bar{a} m)=(v, m, a)+(v, a, m)=0$, and similarly $(m v) a-(a m) v=0$.

Now, let $u \in V_{a}, m \in M, a, b \in H$. Then $(u m) a-(u \bar{a}) m=(u, m, a)-(u, \bar{a}, m)+$ $u(m a-\bar{a} m)=0$, and similarly $a(m u)-(\bar{a} u) m=0$, which proves (21) and (22). Furthermore, by (21), $(u m) a \cdot b=(u \bar{a} \cdot m) b=(u \bar{a} \cdot \bar{b}) m=(u \cdot \overline{b a}) m=(u m)(b a)$, which proves (23). Similarly, by (22), one gets (24). Finally, (25) and (26) follow easily from (23) and (24). 
Lemma 3.2. Let $V=V_{a} \oplus V_{c}$ be a unital alternative superbimodule over $B(4,2)=$ $H+M$. Then, $V_{a} M, M V_{a}, V_{c} M$ and $M V_{c}$ are $H$-invariant subspaces. Moreover $V_{a} M+M V_{a} \subseteq V_{c}$ and $V_{c} M+M V_{c} \subseteq V_{a}$.

Proof. Since $V_{a}, V_{c}$, and $M$ are $H$-invariant, it suffices to prove, for the first part of the lemma, that the product of any $H$-invariant subspaces $U$ and $W$ in the split extension superalgebra $E=B+V$ is again $H$-invariant.

We have $(U W) H \subseteq U(W H)+(U, W, H) \subseteq U W+(U, H, W) \subseteq U W$, and similarly $H(U W) \subseteq U W$.

Now, let us prove that $V_{a} M+M V_{a} \subseteq V_{c}$. Recall that, by Proposition 3.2 $V_{c}=(V, H, H)$. Choose $a, b \in H$ such that $[a, b]^{2} \neq 0$. Then $0 \neq[a, b]^{2} \in F$, and, by (26),

$$
M V_{a}=[a, b]^{2}\left(M V_{a}\right) \subseteq[a, b]\left(M V_{a}\right) \subseteq\left(a, b, M V_{a}\right) \subseteq(H, H, V)=V_{c},
$$

and similarly $V_{a} M \subseteq V_{c}$.

Finally, for any $v \in V_{c}, m \in M, a \in H$, we have by (19) and (15)

$$
((v m) a) b=((a v) m) b=(b(a v)) m=((a b) v) m=(v m)(a b),
$$

which proves that $V_{c} M \subseteq V_{a}$. Similarly, by (20) and (15), $M V_{c} \subseteq V_{a}$.

Corollary 3.1. In the notation of the lemma, $V_{a} \neq 0$.

Really, if $V_{a}=0$, then $V=V_{c}$ and $V M=M V=0$, which yields, for any $v \in V$,

$$
v=v \cdot\left(m_{1} m_{2}-m_{2} m_{1}\right)=\left(v m_{1}\right) m_{2}-\left(v m_{2}\right) m_{1}=0,
$$

a contradiction.

Lemma 3.3. Let $V$ be a unital alternative superbimodule over $B=B(4,2)=$ $H+M$, and let $Z_{a}=Z_{a}(V)=\left\{v \in V_{a} \mid[v, H]=0\right\}$. Then, $Z_{a} \neq 0$ and satisfies the following conditions:

i) $\left[Z_{a}, B\right]=0$,

ii) $\left(Z_{a}, B, B\right)=0$.

Proof. By Corollary 3.1, $V_{a}$ is a nonzero unital bimodule over $H$. The category of unital $H$-bimodules is equivalent to the category of right unital $H^{\circ} \otimes H$-modules [4], where $H^{\circ}$ is the algebra anti-isomorphic to $H$. Since $H^{\circ} \otimes H \cong M_{4}(F)$, this means that every unital $H$-bimodule is completely reducible and that any two unital irreducible $H$-bimodules are isomorphic. The regular $H$-bimodule $\operatorname{Reg} H$ is unital and irreducible; therefore, the bimodule $V_{a}=\bigoplus_{i} W_{i}$, where each $W_{i}$ is isomorphic to $\operatorname{Reg} H$. It is now clear that $Z_{a} \neq 0$.

Let us prove first that

$$
\left(Z_{a}, H, M\right)=0 \text {. }
$$

By Lemma 3.2 for any $u \in Z_{a}, a \in H, m \in M$ we have $(a, u, m)=(a u) m-a(u m) \stackrel{\text { 14 }}{=}(a u) m-(u m) \bar{a} \stackrel{\text { 21] }}{=}(a u) m-(u a) m=[a, u] m=0$, which proves (27). Furthermore, consider the identity

$$
([x, y], y, z)=[y,(x, y, z)],
$$

which holds in any alternative algebra. Using its superized linearization, we have for any $u \in Z_{a}, m \in M, a, b \in H$

$$
([u, m], a, b)=-([u, a], m, b)+(-1)^{d(m) d(u)}[m,(u, a, b)]+[a,(u, m, b)]=0,
$$


since $[u, a]=(u, a, b)=0$ and $(u, m, b)=0$. Therefore, $\left(\left[Z_{a}, M\right], H, H\right)=0$.

By (15),

$$
0=([u, m], a, b)=([u, m] a) b-[u, m](a b)=[u, m](b a)-[u, m](a b)=[u, m][b, a] .
$$

Therefore, $\left[Z_{a}, M\right][H, H]=0$, which yields $\left[Z_{a}, M\right]=0$, proving $i$ ).

Consider now the identity

$$
2[(x, y, z), t]=([x, y], z, t)+([y, z], x, t)+([z, x], y, t),
$$

which holds in every alternative algebra (see 7], Lemma 3.2). Using the corresponding superidentity, we have for any $u \in Z_{a}, m, n \in M, a \in H$,

$$
2[(u, m, n), a]=([u, m], n, a)+([m, n], u, a)-(-1)^{d(u)}([n, u], m, a)=0,
$$

by $i$ ) and (27). Therefore, $\left[\left(Z_{a}, M, M\right), H\right]=0$, and by superized linearization of (28) we have

$$
0=[a,(u, m, n)]=-(-1)^{d(u)}[m,(u, a, n)]+(u, m,[n, a])-(u, a,[n, m]) .
$$

By (27) and the fact that $Z_{a} \subseteq V_{a}$, this implies the equality $\left(Z_{a}, M,[M, H]\right)=0$. But it is easy to see that $[M, H]=M$; hence $\left(Z_{a}, M, M\right)=0$, yielding $\left.i i\right)$.

Proof of Theorem 3.1. Let $V=V_{a} \oplus V_{c}$ be a unital irreducible alternative superbimodule over $B=B(4,2)=H+M$. By Lemma 3.3, $Z_{a} \neq 0$; so we can choose some homogeneous element $0 \neq u \in Z_{a}$. The conditions $i$ ) and $i i$ ) of Lemma 3.3 show that the subspace $u \cdot B$ is a $B$-subbimodule of $V$ and the mapping $\varphi: a \mapsto u \cdot a$ is a $B$-bimodule homomorphism of $\operatorname{Reg} B$ onto $u B$, in the case where $u$ is even, or of $(\operatorname{Reg} B)^{o p}$ onto $u B$, in the case where $u$ is odd. Since both $\operatorname{Reg} B$ and $(\operatorname{Reg} B)^{o p}$ are irreducible, and $\varphi(1)=u \neq 0$, we have that $u B=V$ is isomorphic to $\operatorname{Reg} B$ or to $(\operatorname{Reg} B)^{o p}$.

Proof of Theorem 3.2. Let $U=U_{a}+U_{c}$ be a unital superbimodule over $B=$ $B(4,2)=H+M$. It was shown in the proof of Lemma 3.3 that the bimodule $U_{a}$ is isomorphic to a direct sum of regular $H$-bimodules: $U_{a}=\bigoplus_{i} U_{i}$, where, for every $i, U_{i}=u_{i} H$, and $u_{i} \in Z_{a}\left(U_{i}\right)$ is the image of the unit 1 under the isomorphism of Reg $H$ onto $U_{i}$. In particular, [ $\left.u_{i}, H\right]=0$; hence, by Lemma 3.3, $u_{i} \in Z_{a}(U)$.

Consider $W=\sum_{i} u_{i} B$. Evidently, $W$ is a $B$-subbimodule of $U$ and $U_{a} \subseteq W$. Let $v \in U_{c}$. Then $v=v\left(m_{1} \circ m_{2}\right)=\left(v m_{1}\right) m_{2}-\left(v m_{2}\right) m_{1}$. By Lemma 3.2 $v m_{i} \in U_{a} \subseteq W$; so $v \in W$ as well, and $U=W$. Since every bimodule $u_{i} \cdot B$ is irreducible, $U=W$ is completely reducible.

\section{Bimodules With SUPERINVOLUTION}

Recall that a linear even mapping $*: A \longrightarrow A$ is called a superinvolution of a superalgebra $A$, if it satisfies the conditions

$$
\left(a^{*}\right)^{*}=a, \quad(a b)^{*}=(-1)^{d(a) d(b)} b^{*} a^{*},
$$

for any homogeneous elements $a, b \in A$.

Now, let $V$ be a superbimodule over a superalgebra $(A, *)$ with superinvolution. By analogy with the non-graded case (see [2]), we will call $V$ an A-bimodule with superinvolution, if there exists a linear mapping $-: V \longrightarrow V$ such that the mapping

$$
a+v \mapsto a^{*}+\bar{v}
$$


is a superinvolution of the split null extension superalgebra $E=A+V$. Evidently, for a superalgebra with superinvolution $A$, the bimodules $\operatorname{Reg} A$ and $(\operatorname{Reg} A)^{o p}$ have the superinvolutions induced by that of $A$.

It was shown in [5] that the superalgebras $B(1,2)$ and $B(4,2)$ admit the following superinvolutions:

In $B(1,2), a_{0}+a_{1} \mapsto a_{0}-a_{1}$; and in $B(4,2), a_{0}+a_{1} \mapsto \overline{a_{0}}-a_{1}$, where the mapping $a \mapsto \bar{a}$ is the symplectic involution of the matrix algebra $M_{2}(F)$.

Now, we will study the structure of superbimodules with superinvolution over $B(1,2)$ and $B(4,2)$. Our first objective is to prove that every irreducible superbimodule with superinvolution over these superalgebras is of the type Reg $B$ or $(\operatorname{Reg} B)^{o p}$.

In fact, we will consider the superbimodules with involution that satisfy the additional condition of so-called $J$-admissibility (see [2]). A superbimodule with superinvolution $(V,-)$ over a superalgebra with superinvolution $(A, *)$ is called $J$ admissible if all the symmetric elements of the superalgebra with superinvolution $E=A+V$ lie in the associative center (the nucleus) of $E$. In fact, only $J$-admissible bimodules are needed for applications to Jordan algebras.

Theorem 4.1. Every irreducible unital $J$-admissible superbimodule $V$ with superinvolution over $B=B(1,2)$ is isomorphic to $\operatorname{Reg} B$ or to $(\operatorname{Reg} B)^{o p}$.

Proof. Let $V$ be a superbimodule under consideration, with a superinvolution $v \mapsto$ $\bar{v}$. Observe first that for any $a \in B, v \in V$, we have

$$
\overline{[a, v]}=\overline{a v}-(-1)^{d(v) d(a)} \overline{v a}=(-1)^{d(a) d(v)} \bar{v} \bar{a}-\bar{a} \bar{v}=-[\bar{a}, \bar{v}] .
$$

This means that the subspace $[V, a]$ is invariant with respect to the superinvolution and so is a subbimodule with superinvolution. Now, all the arguments of the proof of Proposition 2.1 are applied to our case, and we conclude that $V$ is a commutative $B$-supermodule.

It is clear that $V=S y m V \oplus S k e w V$, where, for any $h \in S y m V, k \in S k e w V$, we have $\bar{h}=h, \bar{k}=-k$. Assume first that $\operatorname{Sym} V \neq 0$ and choose some $0 \neq h \in$ Sym $V$. By $J$-admissibility, $(h, B, B)=0$, and so we have

$$
\begin{aligned}
& (h x) x=(h, x, x)+h(x x)=0, \quad(h y) y=0, \\
& (h x) y=(h, x, y)+h(x y)=h(x y)=h, \quad(h y) x=-h, \\
& \overline{h x}=(-1)^{d(h)} \bar{x} \bar{h}=-(-1)^{d(h)} x h=-h x, \quad \overline{h y}=-h y .
\end{aligned}
$$

Therefore, the subspace $U=F h+F(h x)+F(h y)$ is a $B$-subbimodule with involution of $V$, and hence $U=V$. It is clear that $U \cong \operatorname{Reg} B$ for even $h$, and $U \cong(\operatorname{Reg} B)^{o p}$ for odd $h$.

Now, assume that Sym $V=0$, that is, $\bar{v}=-v$ for any $v \in V$. Then we have

$$
\overline{v x}=(-1)^{d(v)} \bar{x} \bar{v}=(-1)^{d(v)} x v=v x ;
$$

hence $v x \in \operatorname{Sym} V=0$. Similarly, $v y=0$, and finally $v=v(x y-y x)=(v x) y-$ $(v y) x=0$, a contradiction.

Theorem 4.2. Every unital $J$-admissible alternative superbimodule $V$ with superinvolution over the superalgebra $B=B(1,2)$ is completely reducible.

Proof. It suffices to prove that $V$ is a sum of irreducible subbimodules with involution, or, equivalently, that every element $v \in V$ lies in a sum of irreducible 
subbimodules with involution. Assume first that $v=h \in \operatorname{Sym} V$. We know that $(h, B, B)=0$. Now let us show that also $[h, B]=0$. Consider

$$
(x h y) x=(x h \cdot y) x=(x h, y, x)+(x h)(y x)=(y, x, x h)-x h=-x h-x h=x h .
$$

On the other hand,

$$
\begin{aligned}
(x h y) x & =(x \cdot h y) x=x(h y \cdot x)+(x, h y, x)=-x h+(-1)^{d(h)}(h y, x, x) \\
& =-x h-(-1)^{d(h)} h x .
\end{aligned}
$$

Hence, $[x, h]=x h-(-1)^{d(h)} h x=0$. Similarly, $[y, h]=0$, and so $[B, h]=0$.

We can now apply the arguments from the proof of Theorem 4.1 which show that the elements $h, h x, h y$ span an irreducible subbimodule with involution of $V$. So, in this case we are done.

Now, let $v=k \in S k e w V$. By the previous arguments, the subbimodule $(\operatorname{Sym} V) B$ generated by symmetric elements of $V$ is completely reducible; so it suffices to prove that $k \in(\operatorname{Sym} V) B$. Below, for $v \in V$ we will write $v \equiv 0$ if $v \in(\operatorname{Sym} V) B$.

It is easy to see that

$$
\text { Skew } V \circ B_{1} \subseteq \text { Sym } V, \quad\left[\text { Skew } V, B_{1}\right] \subseteq \text { Skew } V
$$

hence $k \circ z \equiv 0$ for any $z \in B_{1}$. Moreover, we have

$$
\begin{aligned}
0 & \equiv(k \circ z) z=k z \cdot z+(-1)^{d(k)} z k \cdot z=(k, z, z)+(-1)^{d(k)} z k \cdot z \\
& =-(-1)^{d(k)}(z, k, z)+(-1)^{d(k)} z k \cdot z=(-1)^{d(k)} z \cdot k z .
\end{aligned}
$$

Linearizing this relation on $z$, we have

$$
x \cdot k y+y \cdot k x \equiv 0 .
$$

Now, consider the element $(k \circ x) y \in\left(\right.$ Skew $\left.V \circ B_{1}\right) B_{1} \subseteq(\operatorname{Sym} V) B_{1}=(\operatorname{Sym} V) \circ$ $B_{1} \subseteq$ Skew $V$. We have

$$
(k \circ x) y=k+(k, x, y)+(-1)^{d(k)} x k \cdot y .
$$

Since the elements $k,(k, x, y),(k \circ x) y$ are skewsymmetric, so is $x k \cdot y$. We have

$$
\overline{x k \cdot y}=(-1)^{d(x) d(k)+d(x) d(y)+d(y) d(k)} \bar{y} \cdot \bar{k} \bar{x}=y \cdot k x ;
$$

hence

$$
x k \cdot y=-y \cdot k x .
$$

Comparing this relation with (31), we get

$$
x k \cdot y=-y \cdot k x \equiv x \cdot k y,
$$

which yields $(x, k, y) \equiv 0$. Now, we have by (30),

$$
k=k \cdot x y \equiv k x \cdot y \equiv \frac{1}{2}[k, x] y \equiv \frac{1}{4}[[k, x], y]=[[k, x], y] .
$$

By Corollary 2.1, for any $B$-superbimodule $V$, the equality $[[[V, B], B], B]=0$ holds. Therefore, we have

$$
k \equiv[[k, x], y] \equiv[[[[k, x], y], x], y]=0,
$$

which proves the theorem.

Corollary 4.1. Every unital alternative $J$-admissible superbimodule with superinvolution over the superalgebra $B(1,2)$ is commutative. 
Now, we turn to bimodules with superinvolution over $B(4,2)$.

Theorem 4.3. Every unital $J$-admissible superbimodule with superinvolution $V$ over the superalgebra with superinvolution $B=B(4,2)$ is completely reducible and is a direct sum of irreducible bimodules with superinvolution isomorphic to Reg $B$ or to $(\operatorname{Reg} B)^{o p}$.

Proof. By Theorem $3.2 V=\bigoplus_{i} B u_{i}$ for certain elements $u_{i} \in Z_{a}=Z_{a}(V)$. In particular, we always have $Z_{a} \neq 0$. Let us show that $Z_{a} \subseteq S y m V$. First, it is easy to see that $Z_{a}$ is invariant under the superinvolution; so $Z_{a}=\left(\operatorname{Sym} V \cap Z_{a}\right) \oplus$ $\left(\right.$ Skew $\left.V \cap Z_{a}\right)$. Assume that there exists $0 \neq u \in Z_{a}$ such that $\bar{u}=-u$. Consider the element $s=u m_{1}=\frac{1}{2} u \circ m_{1}$ (recall that $[u, B]=0$ ), where $m_{1}$ is one of the two basic elements of $M$. It is easy to check that $\bar{s}=s$; hence, by $J$-admissibility of $V$, we should have $(s, B, B)=0$. But, by Lemma $3.3\left(u m_{1}, m_{2}, m_{1}\right)=-u m_{1}$. Hence $s=0$, a contradiction.

Now, if $V$ is irreducible then, for any homogeneous $0 \neq u \in Z_{a}$ we have $V=u B$, which is isomorphic to $\operatorname{Reg} B$ or to its opposite, according to the parity of $u$, under the isomorphism $b \mapsto u b$.

In the general case, it suffices to notice that every $u_{i}$ generates an irreducible subsuperbimodule which is invariant under the superinvolution and is isomorphic to $\operatorname{Reg} B$ or to its opposite.

\section{FACTORization theOREMS}

In this section, we will prove for the superalgebras $B(1,2)$ and $B(4,2)$ some analogue of the Kronecker factorization theorem for Cayley algebras from [3].

Theorem 5.1. Let $B$ be an alternative superalgebra with $J$-admissible superinvolution (that is, every symmetric element lies in the nucleus of $B$ ) such that $B$ contains $B(1,2)$ as a unital subsuperalgebra with superinvolution. Then $B \cong U \widetilde{\otimes} B(1,2)$ for a certain commutative associative superalgebra $U$, where $\widetilde{\otimes}$ denotes a graded tensor product, that is,

$$
(u \widetilde{\otimes} a)(v \widetilde{\otimes} b)=(-1)^{d(a) d(v)}(u v) \widetilde{\otimes}(a b)
$$

for any homogeneous $u, v \in U, a, b \in B(1,2)$. In particular, the superalgebra $B$ is commutative.

Proof. Consider $B$ as a $B(1,2)$-superbimodule with superinvolution. By Theorem 4.2 and $J$-admissibility, we conclude that $B=\sum_{i} u_{i} B(1,2)$, where $\overline{u_{i}}=$ $u_{i},\left(u_{i}, B, B\right)=0$. Moreover, $[B, B(1,2)]=0$, by Corollary 4.1 Let $U=\operatorname{Sym} B=$ $\{u \in B \mid \bar{u}=u\}$. Then $B=U B(1,2)$, and we will show that this product is isomorphic to the tensor product we are looking for.

Consider the following identity, which is valid in any alternative algebra (see [7]):

$$
[a, b](a, b, c)-(a, b,(a, b, c))=0 .
$$

Superlinearizing it, we have for any $u, v \in U, a, b, c \in B(1,2)$

$$
\begin{aligned}
{[u, v](a, b, c)=} & \pm[a, v](u, b, c) \pm[u, b](a, v, c) \pm[a, b](u, v, c) \pm(u, v,(a, b, c)) \\
& \pm(a, v,(u, b, c)) \pm(u, b,(a, v, c)) \pm(a, b,(u, v, c))=0 .
\end{aligned}
$$

It is easy to see that $(B(1,2), B(1,2), B(1,2))=(B(1,2))_{1}=F x+F y$; hence $[u, v] x=[u, v] y=0$ and $[u, v]=-[u, v](x y-y x)=-([u, v] x) y+([u, v] y) x=0$. 
Therefore, $[U, U]=0$. Since $U \circ U \subseteq U$, this proves that $U$ is a commutative (and associative) subsuperalgebra of $B$.

Furthermore, we have for any $u, v \in U, a, b \in B(1,2)$,

$$
\begin{aligned}
(u a)(v b) & =u(a v b)=u\left([a, v] b+(-1)^{d(a) d(v)} v a b\right)=(-1)^{d(a) d(v)} u(v a b) \\
& =(-1)^{d(a) d(v)}(u v)(a b),
\end{aligned}
$$

which shows that $B$ is a homomorphic image of $U \widetilde{\otimes} B(1,2)$. Assume that $u+$ $v x+w y=0$ for some $u, v, w \in U$. Then $u \in \operatorname{Sym} B, v x+w y \in$ Skew $B$; hence $u=v x+w y=0$. Moreover, we have $0=(v x+w y) x=-w$ and $0=(v x+w y) y=v$. Therefore, $B \cong U \widetilde{\otimes} B(1,2)$.

One can easily see that, since $U$ and $B(1,2)$ are commutative superalgebras, so is $B$.

Theorem 5.2. Let $B$ be an alternative superalgebra such that $B$ contains $B(4,2)$ as a unital subsuperalgebra. Then $B \cong U \widetilde{\otimes} B(4,2)$ for a certain commutative associative superalgebra $U$.

Proof. As before, consider $B$ as a $B(4,2)$-superbimodule. By Theorem 4.3 . $B=$ $\sum_{i} u_{i} B(4,2)$, where $u_{i} \in Z_{a}(B)=\{u \in B \mid[u, B(4,2)]=0\}$. Set $U=Z_{a}$. Then $B=U B(4,2)$, and we will show that $U$ is the desired superalgebra.

Let us see first that $U$ is a subsuperalgebra of $B$. Fix arbitrary $u, v, w \in$ $U, a, b, c \in B(4,2)$. Then, by (3),

$$
[u v, a]=u[v, a]+(-1)^{d(v) d(a)}[u, a] v=0 ;
$$

hence $U U \subseteq U$. Furthermore, by Lemma 3.3. $(U, B(4,2), B(4,2))=0$, and so, by superization of (29),

$$
([a, b], u, v)= \pm([b, u], a, v) \pm([u, a], b, v) \pm[(a, b, u), v]=0 .
$$

Since $B(4,2)=F 1+[B(4,2), B(4,2)]$, this yields that $(U, U, B(4,2))=0$.

Furthermore, by superized linearization of (33), we have

$$
\begin{aligned}
{[a, b](u, v, w)=} & \pm[a, v](u, b, w) \pm[u, b](a, v, w) \pm[u, v](a, b, w) \pm(a, b,(u, v, w)) \\
& \pm(a, v,(u, b, w)) \pm(u, b,(a, v, w)) \pm(u, v,(a, b, w))=0 .
\end{aligned}
$$

Choose $a, b \in B(4,2)_{0}=M_{2}(F)$ such that $[a, b]^{2}=\alpha \in F, \alpha \neq 0$. Then $\alpha(u, v, w)=[a, b]^{2}(u, v, w)=[a, b]([a, b](u, v, w))=0$ and $(u, v, w)=0$. Thus, $U$ is associative.

Applying again the superized linearization of (33), we get

$$
\begin{aligned}
{[u, v](a, b, c)=} & \pm[a, v](u, b, c) \pm[u, b](a, v, c) \pm[a, b](u, v, c) \pm(u, v,(a, b, c)) \\
& \pm(a, v,(u, b, c)) \pm(u, b,(a, v, c)) \pm(a, b,(u, v, c))=0 .
\end{aligned}
$$

Since $m_{i}=-\left(e_{i i}, e_{j i}, m_{j}\right), i, j=1,2, i \neq j$, this implies $[u, v] m_{i}=0, i=1,2$, and finally

$$
[u, v]=[u, v]\left(m_{1} m_{2}-m_{2} m_{1}\right)=\left([u, v] m_{1}\right) m_{2}-\left([u, v] m_{2}\right) m_{1}=0 .
$$

Therefore, $U$ is a commutative and associative subsuperalgebra of $B$.

It is clear that $B$ is a homomorphic image of $U \widetilde{\otimes} B(4,2)$. Assume that $w=$ $\sum_{i j} u_{i j} e_{i j}+u_{1} m_{1}+u_{2} m_{2}=0$ for some $u_{i}, u_{i j} \in U$. Then we have

$$
\begin{aligned}
& 0=\left(e_{11}, e_{21}, w\right)=-u_{2} m_{1}, \\
& 0=\left(e_{22}, e_{12}, w\right)=-u_{1} m_{2},
\end{aligned}
$$


which implies easily that $u_{1}=u_{2}=0$. Furthermore,

$$
0=\left(e_{i i} w\right) e_{j j}=u_{i j} e_{i j},
$$

which yields easily $u_{i j}=0$ for all $i, j$.

\section{REFERENCES}

1. S.González, M.C.López-Díaz, C.Martínez, and I.P.Shestakov, Bernstein Superalgebras and Supermodules, J. of Algebra, 212 (1999), 119-131. MR 99g:17056

2. N.Jacobson, Structure and Representations of Jordan Algebras, Amer. Math. Soc. Colloq. Publ., Vol. XXXIX, Amer. Math. Soc., Providence, RI, 1968. MR 40:4330

3. N.Jacobson, A Kronecker factorization theorem for Cayley algebras and the exceptional simple Jordan algebras, Amer. J. Math., 76 (1954), 447-452. MR 15:774c

4. R.S.Pierce, Associative Algebras, Springer-Verlag, New York, 1982. MR 84c:16001

5. I.P.Shestakov, Prime alternative superalgebras of arbitrary characteristic, Algebra i Logika, 36, No. 6 (1997), 675-716; English transl.: Algebra and Logic 36, No. 6 (1997), 389-420. MR 99k:17006

6. E.I.Zelmanov and I.P.Shestakov, Prime alternative superalgebras and nilpotence of the radical of a free alternative algebra, Izv. Akad. Nauk SSSR Ser. Mat. 54 (1990), 676-693; English transl. in Math.USSR Izv. 37 (1991), 1, 19-36. MR 91j:17003

7. K.A.Zhevlakov, A.M.Slin'ko, I.P.Shestakov, A.I.Shirshov, Rings that are nearly associative, Nauka, Moscow, 1978; English transl., Academic Press, 1982. MR 83i:17001

Departamento de Matemáticas, Universidad de Oviedo, C/ Calvo Sotelo, s/n, 33007, OVIEDO, SPAIN

E-mail address: cld@pinon.ccu.uniovi.es

Instituto de Matemática e Estatística, Universidade de São Paulo, Caixa Postal 66281 - CEP 05315-970, SÃo PAulo, Brasil

Sobolev Institute of Mathematics, Novosibirsk, 630090, Russia

E-mail address: shestak@ime.usp.br 